Z Herz- Thorax- Gefäßchir 2021 · 35:344-345 https://doi.org/10.1007/s00398-021-00462-x Angenommen: 29. September 2021

(c) Springer Medizin Verlag $\mathrm{GmbH}$, ein Teil von Springer Nature 2021

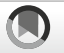

\section{Autor}
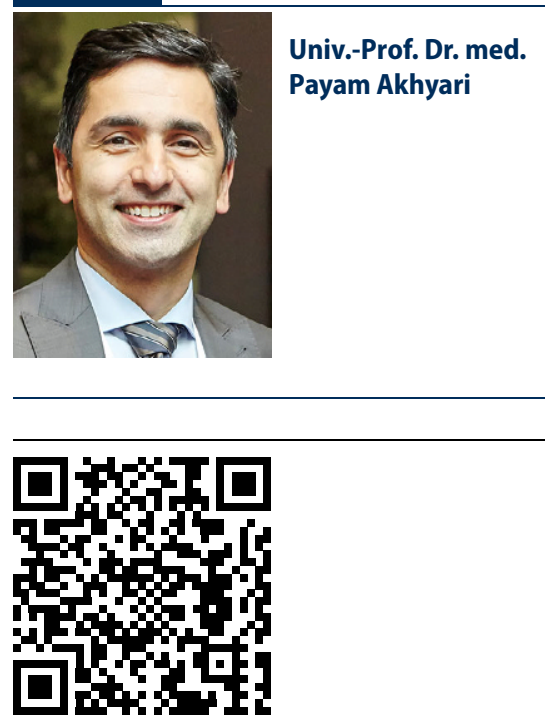

QR-Code scannen \& Beitrag online lesen

\title{
Neue Rubrik: Handlungsalgorithmen
}

\author{
Payam Akhyari \\ Klinik für Herzchirurgie, Universitätsklinikum Düsseldorf, Düsseldorf, Deutschland
}

Liebe Leserinnen und Leser,

die Therapiepfade, mit denen sich die Deutsche Gesellschaft für Herz-, Thoraxund Gefäßchirurgie als wissenschaftliche Fachgesellschaft beschäftigt, haben in den vergangenen Jahren einen erheblichen Wandel durchlaufen. Dies betrifft in erster Linie die Therapie kardialer Erkrankungen, jedoch in abgestufter Form ebenso thoraxchirurgische sowie gefäßchirurgische Therapiepfade. Die damit einhergehenden Veränderungen für unsere klinische Praxis werden in regelmäßigen Intervallen einer wissenschaftlichen Überprüfung und Anpassung unterzogen, deren Ergebnisse sich in den Leitlinien unserer Fachgesellschaften wiederfinden, in der Regel ausgehend von den Leitlinien der europäischen Fachgesellschaften, z. B. der European Society for Cardiology (ESC) und European Association for CardioThoracic Surgery (EACTS). Diese gemeinschaftlich erstellten Publikationen beinhalten in recht umfangreichen Schriften nicht nur die zahlreichen evidenzbasierten Empfehlungssätze. Das Studium der Leitlinienveröffentlichungen bietet auch die Möglichkeit, die wissenschaftliche Grundlage für die Erteilung der jeweiligen Empfehlungen im Einzelnen nachvollziehen zu können. Diesem hohen Anspruch an wissenschaftlicher Aufarbeitung und ebenso dem Wunsch nach umfassender Gültigkeit in praktisch allen klinischen Situationen geschuldet sind jedoch die Leitlinien in ihrer Originalfassung von großem Umfang. Sowohl die evidenzbasierte medizinische Patientenversorgung als auch die kompetente Vertretung der eigenen Fachrichtung in interdisziplinären Fallbesprechungen (Heart-Team-Besprechung, Tumorboard, Endokarditis-Board,
Aorten-Board etc.) erfordern das Studium der Veröffentlichungen zu den aktuellen Leitlinien in ihrer Originalform. Gleichwohl sind die Originalpublikationen zu den Leitlinien aus oben genannten Gründen für die tägliche Praxis grundsätzlich nicht ideal, v.a. aufgrund der nichthinreichenden Übersichtlichkeit.

Vor diesem Hintergrund wurde jüngst die Rubrik "Handlungsalgorithmen“ als Teil der Zeitschrift für Herz-, Thorax- und Gefäßchirurgie ins Lebens gerufen, um eine kondensierte und auf die wesentlichen Aspekte fokussierte Darstellung relevanter klinischer Abläufe aus der Praxis der Herz-, Thorax- und Gefäßchirurgie zu bieten. Naturgemäß bewegt sich die Rubrik "Handlungsalgorithmen" inhaltlich einerseits zwischen einer Minizusammenfassung aktueller wissenschaftlicher Empfehlungen und andererseits Vorgehensweisen oder auch Expertenmeinungen, die aus der etablierten klinisch Praxis abgeleitet sind und deren wissenschaftliche Evidenz noch einer Überprüfung bedarf. Die Beiträge dieser Rubrik sind eng an praktisch-klinischen Überlegungen orientiert und stellen dabei einen Vorschlag der Autorinnen und Autoren für die strategische Vorgehensweise dar, der durchaus einer Diskussion und fortlaufenden Aktualisierung ausgesetzt sein kann. Folgerichtig sind die Inhalte dieser Rubrik in Abgrenzung zu den Leitlinien unserer Fachgesellschaften als eine primär strategische Anleitung zu verstehen, jedoch nicht als eine rechtlich bindende Vorgabe. Die Beiträge werden auf einer Doppelseite kondensiert präsentiert, wobei Sie auf der linken Seite die wesentlichen Informationen in Stichworten wiederfinden, während die gegenüberliegende Seite eine grafische Zusammenfassung beinhaltet. 
Als Rubrikleiter wurde mir vom Herausgeberkreis der Zeitschrift für Herz-, Thorax- und Gefäßchirurgie die ehrenvolle Aufgabe zugetragen, die Auswahl der Themen und der Autorinnen und Autoren sowie die Sichtung der Beiträge vorzunehmen. In dieser Funktion bin ich für Initiativeinreichungen aus der Leserschaft der Zeitschrift für Herz-, Thoraxund Gefäßchirurgie dankbar und möchte Sie gerne hierzu ermuntern. Zusammen mit den übrigen Rubrikenleitungen der Zeitschrift für Herz-, Thorax- und Gefäßchirurgie wünschen wir Ihnen eine erkenntnisreiche und kurzweilige Lektüre des ersten Beitrages von Frau PD Dr. Verena Veulemans, der sich mit der Nachsorge und der möglichen Behandlung der residuellen Mitralklappeninsuffizienz nach Aortenklappenersatz beschäftigt.

Mit herzlichen Grüßen

Ihr Payam Akhyari

\section{Korrespondenzadresse}

Univ.-Prof. Dr. med. Payam Akhyari

Klinik für Herzchirurgie, Universitätsklinikum Düsseldorf

Moorenstr. 5, 40225 Düsseldorf, Deutschland payam.akhyari@med.uni-duesseldorf.de

Interessenkonflikt. P. Akhyari gibt an, dass kein Interessenkonflikt besteht.

\section{Hier steht eine Anzeige.}

